\title{
Un análisis bibliométrico de los trabajos libres de psiquiatría presentados a congresos de la Sociedad Chilena de Neurología, Psiquiatría y Neurocirugía entre 2000 y 2009
}

\author{
A bibliometric analysis of free submissions presented \\ to the psychiatry section of the congresses of the \\ Sociedad Chilena de Neurología, Psiquiatría y \\ Neurocirugía, 2000-2009
}

Ramón Florenzano U. ${ }^{1,2}$, Katherine San Juan B. ${ }^{1}$, Carolina Villena C. ${ }^{1,2,3}$, Nicole Taha G. ${ }^{1,3}$ y Andrés Fogar F. ${ }^{1,3}$

\begin{abstract}
Introduction: Bibliometry is the discipline that measures the bibliographic production in a given scientific area; in Chile there are few studies documenting the themes and trends in Mental health research. Method: A quantitative analysis is performed of the 992 papers published in the Abstracts Book in the SONEPSYN meetings between 2000 and 2009. The most productive groups are mentioned, and a classification of main research themes according to the index of the Tratado de Psiquiatría edited by Heerlein et al. Results: The most frequent themes were Affective Disorders, (14,3\%), Liaison and General Hospital Psychiatry (8,6\%), Epidemiology and Classification (6,6\%), Validation and use of diagnostic and psychometric scales (5,6\%), followed by antidepressant use and teaching with 1,2\% each. Most presentations were prepared in university settings (48\%) or in hospitals (35\%), with a strong concentration in the Metropolitan Region (80,4\%) and the VIII Region (8,3\%). Conclusions: There is an increasing number of psychiatric papers presented from 2000 to 2009, with the Metropolitan Region of Santiago the area that present the higher number of research work (76,31\%). There is a low number of collaborative research: $88,3 \%$ of the works were presented by a single institution. 9,6\% (this is 59 papers) were presented by two, and 2,1\% (13 papers) by three. Affective disorders were the most researched area; followed by use of antipsychotics and liaision, community, and child and adolescent psychiatry.
\end{abstract}

Key words: Bibliometry; psychiatric research; psychiatric disorders.

Rev Chil Neuro-Psiquiat 2011; 49 (2): 200-205

Recibido: 28/12/2010

Aprobado 15/03/2011

Servicio de Psiquiatría Hospital del Salvador.

Facultad de Psicología Universidad del Desarrollo.

Psicólogo (a). 


\section{Introducción}

$\mathrm{L}$ a bibliometría es la disciplina que mide la producción bibliográfica en un área científica determinada ${ }^{1}$. En Chile, hay pocos estudios que documenten los temas y tendencias de la producción en Salud Mental. La Sociedad Chilena de Neurología, Psiquiatría y Neurocirugía (SONEPSYN) es la más antigua sociedad científica en el área de la Salud Mental, y su Congreso Anual el más concurrido. Los trabajos libres presentados a los congresos de sociedades científicas representan el modo de publicación más directo y accesible a investigadores y equipos institucionales, ya que las conferencias en esos eventos muchas veces corresponden a peticiones de los organizadores; los posters son publicados habitualmente en ediciones especiales de las revistas, lo que sucede en el caso de SONEPSYN; existen pocos estudios bibliométricos que estudien los trabajos libres como modo de análisis que documente la productividad científica de estos grupos.

En 2004, García-Silversen et $\mathrm{al}^{2}$, hacen un análisis de la producción bibliográfica de la Dirección de Investigaciones Epidemiológicas del Instituto Nacional de Psiquiatría Ramón de la Fuente de México, al cumplir 20 años de existencia. En otros estudios $^{3,4}$ se han estudiado las publicaciones en castellano en el área de la Psicología de la Salud, o bien en las publicaciones en Salud Pública.

El año 2000, Moncada y Olivos ${ }^{5}$, realizan un estudio de las investigaciones realizadas en torno al tema de la psicoterapia entre los años 1990 y 1999, específicamente, qué profesionales han investigado y en qué temas. Esto desde una perspectiva desde la visión del clínico, de las psicoterapias integrativas, de los factores curativos y de la necesidad de pluralismo metodológico. Este estudio encontró que de 216 artículos publicados en diversas revistas entre dichos años, el 22,2\% abordaron el tema del diagnóstico, el 19,9\% epidemiología, el 10,6\% tratamientos y el 9,7\% el abordaje clínico-social. El 56,5\% de los estudios fueron realizados por psiquiatras, el $29,6 \%$ por psicólogos y el $6,5 \%$ por neurólogos. El 68,5\% de las investigaciones fueron realizadas en Santiago, el 12\% en Concepción y el $4,2 \%$ en Valparaíso.
El objetivo de esta presentación es analizar las presentaciones en modalidad de poster en los Congresos Anuales de SONEPSYN entre los años 2000 y 2009, revisando el número anual de publicaciones y su tendencia a lo largo de estos diez años, clasificando los temas y sub-temas de los trabajos, para finalizar con un análisis de la productividad y asociatividad de los diversos grupos.

\section{Metodología}

Se revisaron los libros de programas de los Congresos de SONEPSYN entre 2000 y 2009, así como la publicación de sus resúmenes, identificándose los autores y sus grupos de trabajo. Estos últimos fueron primero agrupados en tres columnas y luego estas se sumaron, preparándose una lista de "asociatividad" que consignaba cuándo los grupos presentaban solos y cuándo lo hacían asociados con una o más instituciones. Se separaron asimismo en presentaciones por grupos universitarios, y por centros asistenciales (hospitales y establecimientos ambulatorios del sistema público, clínicas y otros dispositivos privados). Se clasificaron finalmente por la región geográfica del país a la que pertenecían.

Para la clasificación temática se utilizó la clasificación empleada en el Tratado de Psiquiatría de Heerlein y $\operatorname{cols}^{6,7}$, agrupándolos en seis grandes temas, y luego en 40 sub-temas específicos. Los temas fueron tabulados consensuando la agrupación entre los co-autores. Finalmente se estudió la tendencia cronológica durante los cinco años estudiados solamente para la clasificación temática.

\section{Resultados}

Se analizó un total de 992 trabajos, que se distribuyeron entre los años 2000 y 2008 en porcentajes crecientes, desde 58 posters presentados el año 2000, a 170 el 2009. La distribución se presenta en la Figura 1.

La distribución de trabajos por regiones se concentró en la Región Metropolitana (80,4\%) y en la 

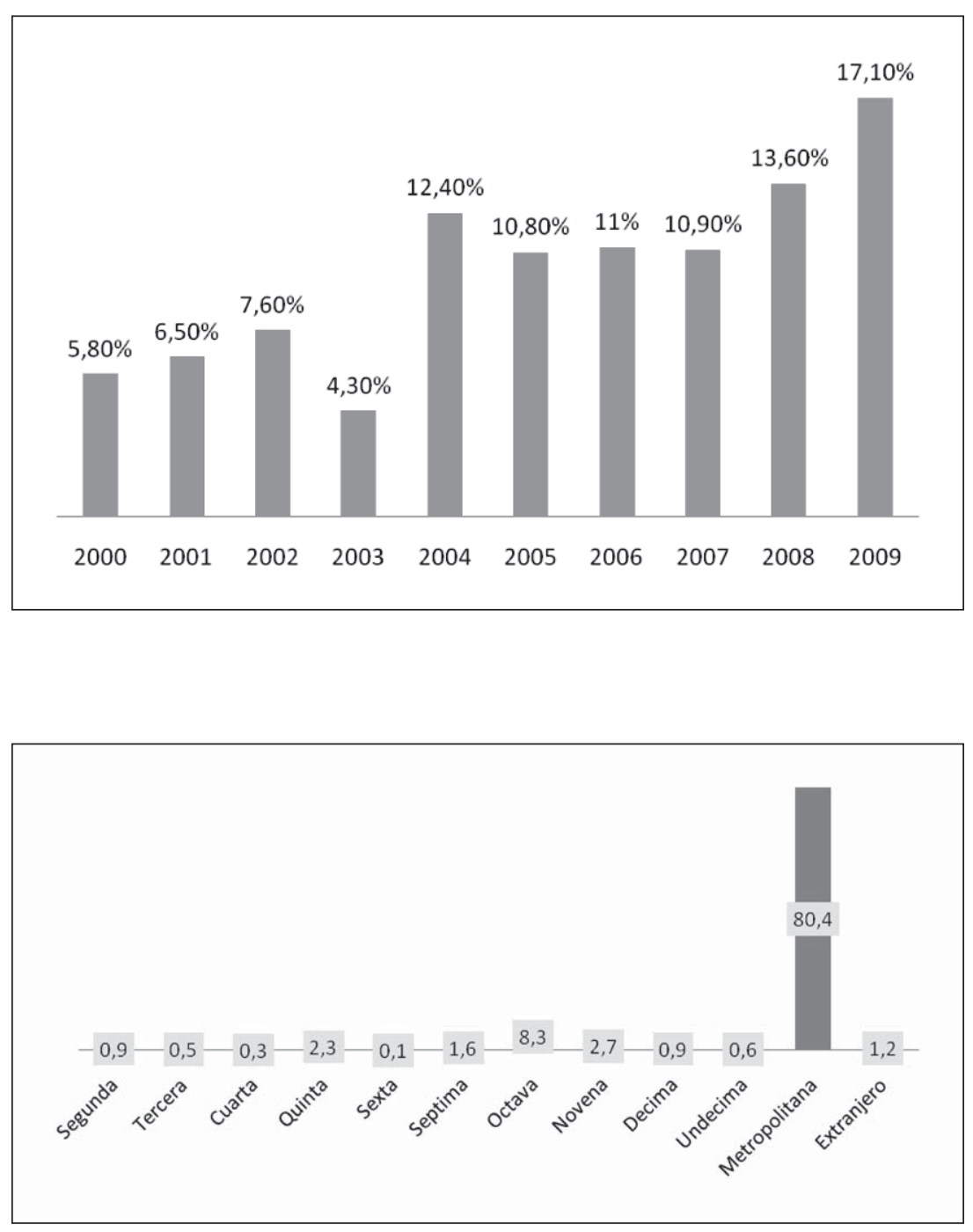

Figura 1. Porcentaje según año de presentaciones en modalidad de pósters, congresos Sociedad Chilena de Neurología, Psiquiatría y Neurocirugía, sección de psiquiatría, 2000-2009.
Figura 2. Distribución por regiones de presentaciones en modalidad de posters, congresos Sociedad Chilena de Neurología, Psiquiatría y Neurocirugía, sección de psiquiatría, 2000-2009.
VIII Región (8,3\%), como se ilustra en la Figura 2. Sólo un $1,2 \%$ de los trabajos procedían de fuera de Chile.

$\mathrm{Al}$ ordenar temáticamente los trabajos, el mayor porcentaje correspondió a Trastornos del ánimo (14,3\%), seguido por Enlace y Psiquiatría de Hospital General $(8,4 \%)$, seguidos por Epidemiologia y Clasificación (6,6\%), Instrumentos de medición $(5,6 \%)$ y Psiquiatría Infanto-Juvenil (5,3\%). Esto se ilustra en la Tabla 1.

$\mathrm{Al}$ ordenar en grandes temas los trabajos, los centrados en características de cuadros clínicos representan un $41,2 \%$, seguidos por trabajos sobre sub-especialidades psiquiátricas (infanto-juvenil, forense, neurociencias). Los temas terapéuticos, sobre todo psicofarmacológicos y psicoeterapéuticos siguieron en frecuencia (10,75\% y 9,53\%).

Casi la mitad de los trabajos (48\%) fueron presentados por grupos universitarios, correspondiendo el siguiente porcentaje a los hospitalarios (35\%). Entre los otros grupos se encontraron en orden decreciente los presentados por autores independientes, sin afiliacion institucional (6,3\%), por centros privados $(4,8 \%)$ y por servicios de salud municipales $(1,7 \%)$.

$\mathrm{Al}$ agrupar los trabajos por Universidades, estas 
Tabla 1. Distribución por temas de 992 trabajos presentados en modalidad póster en los congresos SONEPSYN entre los años 2000 y 2009

\begin{tabular}{|c|c|c|}
\hline Tema principal & Frecuencia & $\%$ \\
\hline Trastornos del ánimo & 142 & 14,3 \\
\hline Enlace y psiquiatría hospital general & 83 & 8,4 \\
\hline Epidemiología y clasificación & 65 & 6,6 \\
\hline Instrumentos de medición & 56 & 5,6 \\
\hline Psiquiatría infanto-juvenil & 53 & 5,3 \\
\hline Esquizofrenia & 52 & 5,2 \\
\hline Antipsicóticos & 49 & 4,9 \\
\hline Conducta suicida & 44 & 4,4 \\
\hline Psiquiatría comunitaria & 40 & 4,0 \\
\hline Alcohol y farmacodependencias & 37 & 3,7 \\
\hline Psiquiatría forense & 35 & 3,5 \\
\hline Investigación en psicoterapia & 34 & 3,4 \\
\hline Trastornos de conducta alimentaria & 34 & 3,4 \\
\hline Trastornos de ansiedad & 24 & 2,4 \\
\hline Cuadros psico-orgánicos & 22 & 2,2 \\
\hline Neurobiología & 22 & 2,2 \\
\hline Trastornos de personalidad & 20 & 2,0 \\
\hline Filosofía y sociología & 19 & 1,9 \\
\hline Emergencias psiquiátricas & 17 & 1,7 \\
\hline Psicofarmacología general & 16 & 1,6 \\
\hline Antidepresivos & 12 & 1,2 \\
\hline Docencia & 12 & 1,2 \\
\hline Otros & 104 & 10,0 \\
\hline Total & 992 & 100 \\
\hline
\end{tabular}

presentaron 456 trabajos a lo largo de los años revisados, con el mayor porcentaje para las diversas sedes de la Universidad de Chile (15,3\%), seguida por la Universidad Católica (14\%), y la de Concepción $(8,8 \%)$. Entre las privadas la que ha presentado un mayor número de trabajos ha sido la Universidad de los Andes (5,7\%), seguida por la del Desarrollo y la de Valparaíso (ambas con 1,8\%). Esto se ilustra en la Tabla 2.

Dentro de los grupos hospitalarios, el mayor número de posters fue presentado por el Instituto Psiquiátrico Dr. José Horwitz Barak, que es el principal establecimiento especializado en la Región Metropolitana y el más antiguo de Chile ( $8,8 \%)$; cercanamente seguido por el Servicio de Psiquiatría del Hospital del Salvador, a su vez el primer establecimiento terciario que contó con una unidad especializada $(8,3 \%)$. En porcentajes menores presentan establecimientos regionales tales como el Hospital de Chillán (2,2\%), el de Curicó (1,5\%), y otros, como se ilustra en la Tabla 3.

$\mathrm{Al}$ analizar la asociatividad en la investigación, se aprecia que el 92,6\% de los trabajos expuestos corresponden a una institución, el $10 \%$ a dos instituciones y sólo el 1,7\% a tres o más.

Tabla 2. Distribución de posters presentados por grupos universitarios, SONEPSYN 2000-2009, por institución

\begin{tabular}{|lcc|}
\hline Universidades & n & \% válido \\
Universidad de Chile (Clínica Psiquiátrica Universitaria y otros) & 153 & 15,3 \\
Pontificia Universidad Católica de Chile (Departamento de Psiquiatría y otros) & 128 & 14,0 \\
\hline Universidad de Concepción (Departamento de Psiquiatría y otros) & 80 & 8,8 \\
Universidad de los Andes & 52 & 5,7 \\
Universidad del Desarrollo & 16 & 1,8 \\
Universidad de Valparaíso & 16 & 1,8 \\
Otras Universidades & 11 & 1,1 \\
\hline
\end{tabular}


Tabla 3. Distribución de posters presentados por grupos hospitalarios, SONEPSYN 2000-2009, por institución

\begin{tabular}{|lcc|}
\hline Hospitales & n & $\begin{array}{c}\% \\
\text { válido }\end{array}$ \\
\hline Instituto Psiquiátrico José Horwitz & 80 & 8,8 \\
$\begin{array}{l}\text { Hospital del Salvador, Servicio de } \\
\text { Psiquiatría y otros }\end{array}$ & 76 & 8,3 \\
Hospital Herminda Martín de & 20 & 2,2 \\
Chillán & & \\
Hospital de Curicó & 14 & 1,5 \\
Hospital del Trabajador & 13 & 1,4 \\
Hospital El Peral & 11 & 1,2 \\
Hospitales Barros Luco Trudeau & 10 & 1,1 \\
Hospital de Temuco & 10 & 2,0 \\
\hline Hospitales FFAA & 9 & 1,0 \\
\hline Hospital de Ovalle & 9 & 1,0 \\
\hline Otros Hospitales & 64 & 7,0 \\
\hline
\end{tabular}

\section{Conclusiones}

Este trabajo presenta publicaciones resumidas de los posters presentados en los Congresos de psiquiatras especialistas, y por lo tanto, no incorpora la productividad de psicólogos y otros profesionales, que ese presentan, por ejemplo, en la Sociedad de Psicólogos Clínicos, de la Sociedad Chilena de Salud Mental, en los Congresos Chilenos de Salud Publica, etc. Sin embargo, hay una coincidencia interesante con el trabajo antes citado de Moncada y Olivos: se puede observar que el porcentaje de estudios abocados a los tratamientos ha sido similar (10,6\% y 10,7\%). Ambos trabajos no son comparables, ya que las temáticas en SONEPSYN son más amplias que el ámbito de la investigación en psicoterapia.

En este trabajo se observa un número creciente de presentaciones desde el año 2000 hasta el 2009. Este pudiera deberse a la modalidad de selección de los trabajos a presentar.

En cuanto a la distribución geográfica, al igual que en el estudio de Moncada y cols, la RM pre- senta el mayor número de investigaciones con un $76,31 \%$, seguido por la VIII Región un 13,62\%. A diferencia de la publicación anterior, la $\mathrm{V}$ región (que incluye Valparaíso) exhibe en este estudio una menor cantidad de trabajos presentados con un $1,68 \%$. Lo anterior muestra como algunas instituciones con tradición en investigación concentran una mayor productividad, así como algunos hospitales de regiones fuera de la Metropolitana, que mantienen una productividad investigativa importante a lo largo de los años.

Hay una baja asociatividad en las presentaciones: un $88,3 \%$ de los trabajos fueron presentados por una sola institución; 9,6\% (o sea 59 trabajos) fueron presentados por dos, y 2,1\% (13 trabajos) por tres instituciones. La recomendación internacional hoy día tiende a realizar trabajos cooperativos, que disminuyen el sesgo de las muestras utiilizadas, y que permite aumentar el tamaño de las poblaciones estudiadas. Son sin embargo, más complejos de organizar y coordinar.

Los temas más frecuentes de los posters presentados son los trabajos psicopatológicos clínicos, seguidos por sub-especialidades psiquiátricas y los referentes a tratamientos. Los trabajos centrados en niños y adolescentes representaron un porcentaje no menor, a pesar de existir otra sociedad (de Psiquiatría del Niño y Adolescente) que realiza también un Congreso Anual. Esto puede ligarse al interés creciente en la prevención de trastornos psicopatológicos del adulto en la edades tempranas.

Los trastornos del ánimo son el sub-tema más analizado, lo que corresponde a la frecuencia de estos cuadros en nuestra población, tanto general como clínica. Este grupo es seguido por antipsicóticos y psiquiatría de enlace, comunitaria e infanto-juvenil. Temas emergentes son evaluación de psicoterapias y de resultados de los tratamiento.

Los grupos universitarios son los más productivos, especialmente los de las Universidades tradicionales. Entre los establecimientos de salud, destacan los servicios cuaternarios y terciarios especializados.La asociatividad es baja: la gran mayoría de los trabajos son presentados por una institución $(88,3 \%)$. 


\begin{abstract}
Resumen
Introducción: La bibliometría es la disciplina que mide la producción bibliográfica en un área científica determinada y en Chile hay pocos estudios que documenten los temas y tendencias de la producción en Salud Mental. Método: Se hace un análisis cuantitativo de los 992 trabajos publicados en los libros de resúmenes de los Congresos entre los años 2000 y 2009. Se describen los grupos más productivos, así como se clasifican los temas de acuerdo a los títulos de los trabajos, al indice del Tratado de Psiquiatría editado por Heerlein y Cols. Resultados: Los temas más frecuentes eran los del trastornos del ánimo (14,3\%), psiquiatría de enlace y hospital general $(8,6 \%)$, epidemiología y clasificación (6,6\%), validación y aplicación de instrumentos diagnósticos $y$ de medición psicológica (5,6\%), y los menos frecuentes antidepresivos y docencia con 1,2\% cada uno. La mayoría de las presentaciones correspondieron a centros universitarios (48\%) u hospitalarios (35\%), con una importante concentración en la Región Metropolitana (80,4\%) y la VIII Región (8,3\%). Conclusiones: Se observa un número creciente de presentaciones desde el año 2000 hasta el 2009, siendo la RM quien presenta el mayor número de investigaciones con un 76,31\%, llama la atención la baja asociatividad en las presentaciones: un $88,3 \%$ de los trabajos fueron presentados por una sola institucion. 9,6\% (o sea 59 trabajos) fueron presentados por dos, y 2,1\% (13 trabajos) por tres instituciones.Los trastornos del ánimo son el sub-tema más analizado, seguido por antipsicóticos y psiquiatría de enlace, comunitaria e infanto-juvenil.
\end{abstract}

Palabras clave: Bibliometría, investigación psiquiátrica, trastornos psiquiátricos.

\section{Referencias}

1. Lukhonnen T. Bibliometrics and evaluation of research performance. Annals Int Medicine 1990; 22: $145-50$.

2. García Silversen S, Arana P, Infante R, Jiménez A. La investigación sobre aspectos epidemiológicos y psicosociales. Salud Mental 2004; 2 (5): $8-22$.

3. Agudelo D, Bustos-López J, Buela-Casal. Análisis bibliométrico de las revistas relacionadas con psicología de la salud editadas en castellano. Salud Mental 2004; 27 (2): 70-84.
4. Pérez AC, Estrada LJM, Villar-Álvarez F, RebolloRodríguez MJ. Estudio Bibliométrico de los Artículos Originales de la Revista Española de Salud Pública (1992-2000): Indicadores Generales. Rev Esp S Pública 2002; 76 (6): 655-72.

5. Moncada L, Olivos P. ¿Es la variable del terapeuta estudiada en Chile? Reflexiones en torno a las investigaciones empíricas de post-grado en psicoterapia. Revista de Psiquiatría y Salud Mental. Año XX. N³, pp. 25-31. Olivos, P. Rojas G.

6. Heerlein AA, editor. Psiquiatría Clínica. Ediciones de la Sociedad de Neurología Psiquiatría y Neurocirugía. Santiago de Chile 2000.

Correspondencia:

Ramon Florenzano U.

Napoleón 3565 oficina 1401

Las Condes, Santiago.

Fono: 02-203718.

E-mail: rflorenzano@gmail.com 